\title{
Metallicity Evolution of AGNs
}

\author{
T. Nagao ${ }^{1,2}$, R. Maiolino ${ }^{3}$, A. Marconi ${ }^{4}$, \\ K. Matsuoka ${ }^{1}$, and Y. Taniguchi ${ }^{2}$ \\ ${ }^{1}$ Graduate School of Science and Engineering, Ehime University, \\ 2-5 Bunkyo-cho, Matsuyama 790-8577, Japan \\ ${ }^{2}$ Research Center for Space and Cosmic Evolution, Ehime University, \\ 2-5 Bunkyo-cho, Matsuyama 790-8577, Japan \\ ${ }^{3}$ INAF - Osservatorio Astrofisico di Roma, \\ via di Frascati 33, 00040 Monte Porzio Catone, Italy \\ ${ }^{4}$ Dipartimento di Astronomia e Scienza dello Spazio, Universita degli Studi di Firenze, \\ Largo E. Fermi 2, 50125 Firenze, Italy
}

\begin{abstract}
Chemical properties of AGNs and their redshift evolution are of interest to understand the star-formation history of AGN host galaxies and the co-evolution between galaxies and supermassive black holes (SMBHs). One important observational clue on this issue is a tight correlation between the AGN luminosity and the metallicity of the broad-line region. Surprisingly this relation shows no redshift evolution, even up to $z \sim 6$. This correlation is attributable mainly to the positive correlation between metallicity and SMBH mass, rather than to the relation between metallicity and Eddington ratio. A significant positive correlation between the metallicity and the AGN luminosity is also seen in narrow-line regions, not only in broad-line regions. Possible implications of these results on the galaxy-SMBH co-evolution are briefly mentioned.
\end{abstract}

Keywords. early universe, galaxies: active, galaxies: evolution, galaxies: high-redshift, galaxies: nuclei, galaxies: Seyfert, quasars: emission lines, quasars: general

\section{Introduction}

Recent observational progress has revealed that nearly all galaxies have a supermassive black hole (SMBH) at their nucleus, at least in the nearby universe. And we now also have observational evidence suggesting that galaxies and SMBHs have experienced so-called "co-evolution." One of the strongest pieces of evidence suggesting this co-evolution is a tight correlation between the mass of the galactic spheroidal component and that of the SMBH (e.g., Marconi \& Hunt 2003), which is hard to understand without some evolutionary link between the two components because their spatial scales differ by an order of magnitude. There is also other observational evidence, including the similar redshift evolution of the number density of massive galaxies and QSOs (e.g., Franceschini et al. 1999), and the AGN-starburst connection (e.g., Imanishi 2002; Silverman et al. 2009). Chemical properties of AGNs have sometimes received attention as another important issue in co-evolution. Metals are actually originally due to the cumulative star-forming activity in the past, so the chemical properties of AGNs should shed light on the issue of co-evolution between SMBHs and galaxies.

The gas-phase metallicity of AGN ionized regions has been studied intensively in the past (see, e.g., Hamann \& Ferland 1999 for a review). A seminal paper by Hamann \& Ferland (1993) reported that QSOs at higher redshift and/or with a higher AGN luminosity $\left(L_{\mathrm{AGN}}\right)$ have a higher metallicity. The presence of QSOs with a super-solar metallicity at high redshift $(z>3)$ immediately suggests that the formation of stars in 
QSO host galaxies occurred quite rapidly in very early universe. However, since we see only high- $L_{\mathrm{AGN}}$ QSOs at high redshifts, it is not clear what primarily determines the QSO gas metallicity, luminosity or redshift. The implication of QSO metallicity studies on the co-evolution of SMBHs and galaxies is also unclear. Motivated by these issues, we have carried out systematic studies on QSO metallicity, both for broad-line regions (BLRs) and narrow-line regions (NLRs).

\section{Metallicity of Broad-Line Regions in SDSS QSOs}

Sometimes the elemental abundance ratio of iron to alpha elements (such as magnesium) is the focus of discussion of the chemical evolution of BLRs in QSOs (e.g., Murayama et al. 1998, 1999; Dietrich et al. 2003a; Maiolino et al. 2003, 2004; Iwamuro et al. $2004)$. This is because iron enrichment requires a moderately long time ( $\sim$ Gyr) and thus its abundance at a high redshift would give strong constraints on the star-formation history of the QSO host galaxies. However, there are large uncertainties and systematic errors in measuring the Fe II multiplet emission and in converting the observed Fe II/Mg II flux ratio to the abundance ratio of Fe/Mg (e.g., Vestergaard \& Wilkes 2001; Sameshima et al. 2009). In addition, accurate measurements of the Fe II multiplet emission require wide wavelength coverage which prevents us from observing Fe II/Mg II flux ratios for high-redshift QSOs.

An alternative approach to investigate BLR metallicity $\left(Z_{\mathrm{BLR}}\right)$ is to focus on the broad Nv $\lambda 1240$ emission line. This is because the nitrogen abundance roughly scales as the square of the metallicity because it is a secondary element. Actually, the flux ratios of $\mathrm{N} \mathrm{V} \lambda 1240 / \mathrm{C}$ IV $\lambda 1549$ and $\mathrm{N} \mathrm{V} \lambda 1240 /$ He II $\lambda 1640$ have often been used to infer

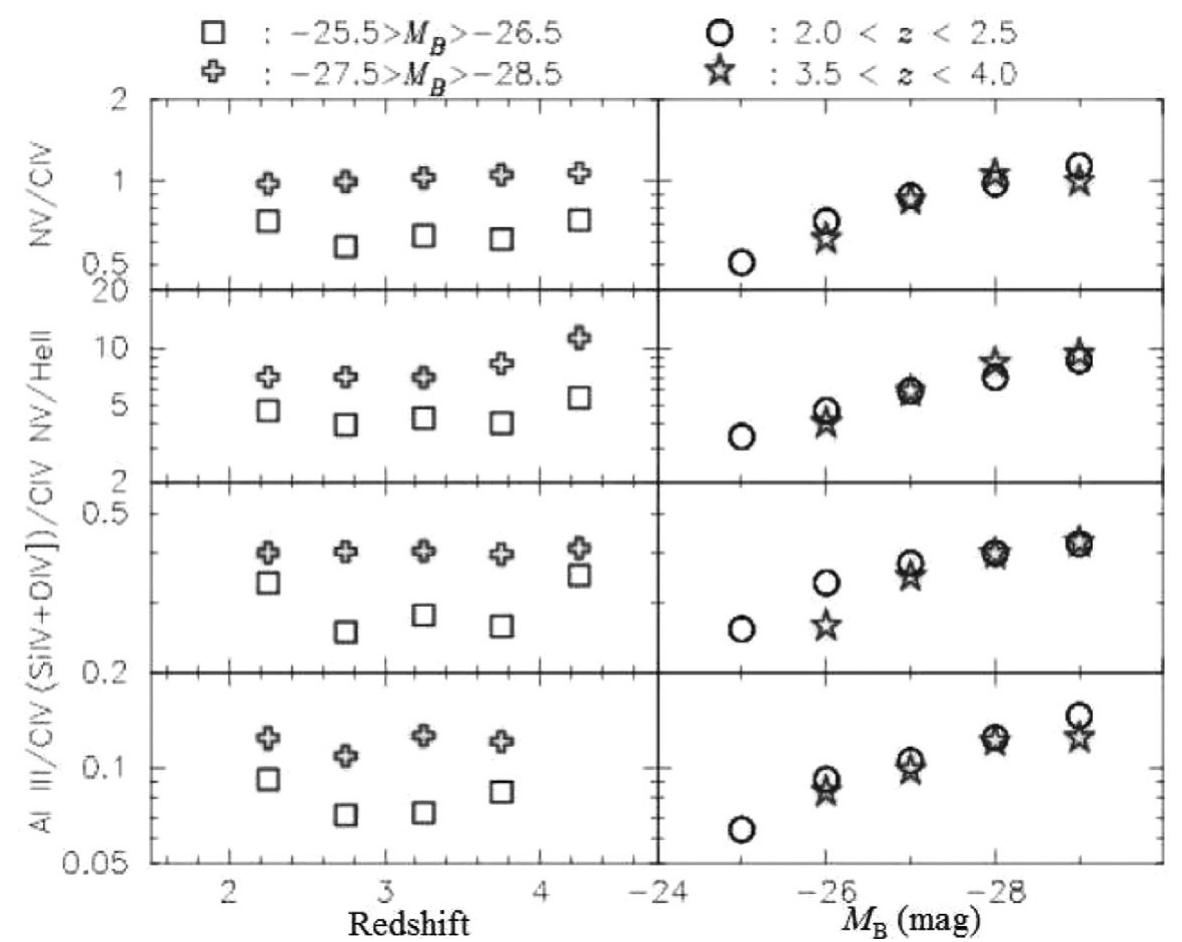

Figure 1. Four metallicity-sensitive emission-line flux ratios of SDSS QSOs, as functions of redshift (left panels) and luminosity (right panels). See Nagao et al. (2006b) for more details. 
$Z_{\text {BLR }}$ (e.g., Hamann \& Ferland 1993; Dietrich et al. 2003b). The measurements of these BLR emission lines are much more straightforward compared to the mesurement of Fe II multiplet emission. Another advantage of this approach is the narrowness of the required wavelength coverage, i.e., $1200 \AA \lesssim \lambda_{\text {obs }} \lesssim 2000 \AA$, even if Al III $\lambda 1857$ and $\mathrm{C}$ III] $\lambda 1909$ are also used.

In order to examine the dependence of $Z_{\mathrm{BLR}}$ on $L_{\mathrm{AGN}}$ and redshift independently, a large QSO sample with a wide luminosity and redshift range is necessary. For this purpose, we focused on the SDSS spectroscopic database; more specifically, we used $>5000$ spectra of SDSS QSOs at $2.0<z<4.5$ (excluding BAL quasars). We then made composite spectra for various sub-samples, e.g., for each $\left(z, L_{\mathrm{AGN}}\right)$ bin. This allows us to examine "typical" BLR spectra for each $\left(z, L_{\mathrm{AGN}}\right)$ bin with a sufficiently high $S / N$ that it enables us also to use rather weak emission lines such as Al III $\lambda 1857$ (see Nagao et al. $2006 \mathrm{~b}$ for details on the sample selection, the method of making the composite spectra, and spectral fitting).

Four flux ratios, N v/C IV, N v/He II, (Si IV+O IV])/C IV, and Al III/C IV, that are metallicity-sensitive are all correlated very tightly with $L_{\mathrm{AGN}}$, as shown in Figure 1 . On the other hand, these flux ratios are nearly constant in the redshift range of $2.0<z<4.5$, if $L_{\mathrm{AGN}}$ is fixed. This suggests that the redshift $-Z_{\mathrm{BLR}}$ relation found by Hamann \& Ferland (1993) was just due to a Malmquist-like bias, i.e., high $Z_{\mathrm{BLR}}$ in high-redshift QSOs is just due to the bias that we are seeing only high- $L_{\mathrm{AGN}}$ QSOs. Surprisingly, there is no significant evolution in the $L_{\mathrm{AGN}}-Z_{\mathrm{BLR}}$ relation, even up to $z \sim 4.5$. Note that, very

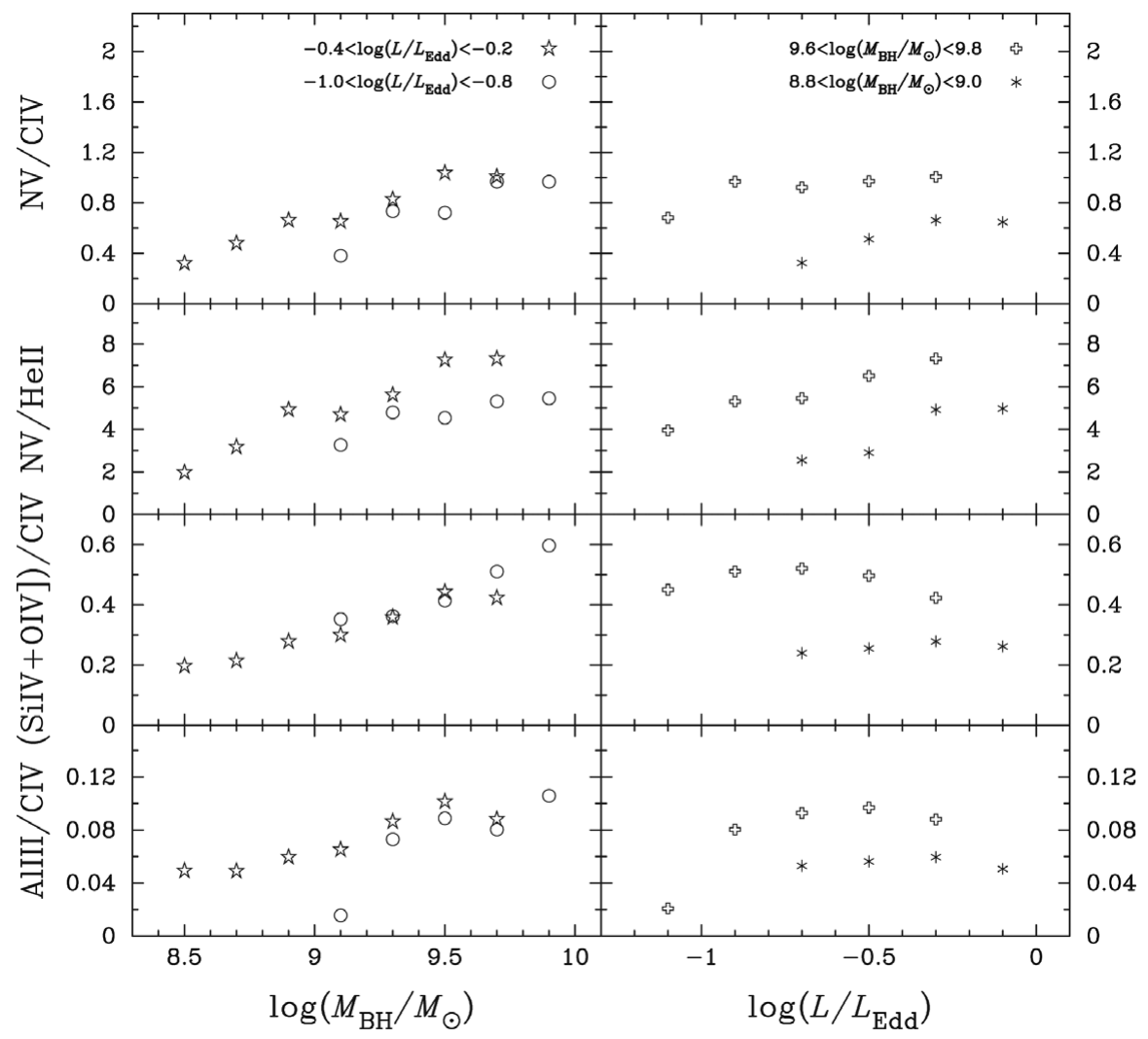

Figure 2. Four metallicity-sensitive emission-line flux ratios, as functions of black-hole mass (left panels) and Eddington ratio (right panels). See Matsuoka et al. (2010) for more details. 
recently, the lack of evolution in the $L_{\mathrm{AGN}}-Z_{\mathrm{BLR}}$ relation has been reported at even much higher redshift, up to $z \sim 6$ (Jiang et al. 2007; Juarez et al. 2009).

Although the clear $L_{\mathrm{AGN}}-Z_{\mathrm{BLR}}$ relation and its lack of evolution up to very high redshift seems to give important constraints on the star-formation history in QSO host galaxies, the discussion is not straightforward. One of the difficulties is that $L_{\mathrm{AGN}}$ is a function of $\mathrm{SMBH}$ mass $\left(M_{\mathrm{BH}}\right)$ and Eddington ratio $\left(L / L_{\mathrm{Edd}}\right)$. Warner et al. $(2004)$ report that the $L_{\mathrm{AGN}}-Z_{\mathrm{BLR}}$ relation is mainly attributed to the $M_{\mathrm{BH}}-Z_{\mathrm{BLR}}$ relation while $Z_{\mathrm{BLR}}$ shows no dependence on $L / L_{\mathrm{Edd}}$. On the other hand, Shemmer et al. (2004) report that $Z_{\mathrm{BLR}}$ depends more significantly on $L / L_{\mathrm{Edd}}$ than $M_{\mathrm{BH}}$. Again the discrimination of these two possibilities requires a large sample with wide parameter ranges. We thus analyzed SDSS composite spectra made for various $\left(M_{\mathrm{BH}}, L / L_{\mathrm{Edd}}\right)$ bins and investigated the dependence of $Z_{\mathrm{BLR}}$ on $M_{\mathrm{BH}}$ and $L / L_{\mathrm{Edd}}$ independently. The results are shown in Figure 2 , in which we can clearly see that $Z_{\mathrm{BLR}}$ depends much more significantly on $M_{\mathrm{BH}}$ than $L / L_{\mathrm{Edd}}$ (see Matsuoka et al. 2010 for more details). We will briefly discuss this result in $\S 4$.

\section{Metallicity of Narrow-Line Regions in Radio Galaxies}

Although the observational study of $Z_{\mathrm{BLR}}$ is relatively easy, in the sense that the BLR emission is strong and easily measured in observed-frame optical spectra of "normal" QSOs at high redshifts, there are some caveats that should be kept in mind. First, the spatial scale of the BLR is very tiny, $r_{\mathrm{BLR}}<1 \mathrm{pc}$, significantly smaller than the spatial scale of host galaxies. It is thus unclear whether $Z_{\mathrm{BLR}}$ is really related to the past starformation history of host galaxies. Second, the total mass of BLR clouds is only $<10 M_{\odot}$ at most, and thus $Z_{\mathrm{BLR}}$ is easily affected by local phenomena such as supernovae in the nuclei. Therefore it would be again unclear whether $Z_{\mathrm{BLR}}$ is a good indicator of the global history of the star formation in host galaxies. One way to reconcile these problems is to focus on NLRs in addition to BLRs. The spatial scale of the NLR is much larger than that of the BLR, and it is rather comparable to that of the host galaxy. Moreover, its total mass is much greater than the BLR total mass. The difficulty in using NLRs is that there in only a relatively small number of known type 2 AGNs in the high-redshift universe, and high-redshift NLR measurements are very difficult in general. Therefore we focus on narrow-line high-redshift radio galaxies (HzRGs) and examine their rest-frame UV emission-line spectra to study $Z_{\mathrm{NLR}}$.

Previously, $Z_{\mathrm{NLR}}$ has been studied using emission-line flux ratios including $\mathrm{N} v \lambda 1240$, as with BLRs (e.g., De Breuck et al. 2000; Overzier et al. 2001). However in NLRs, the $\mathrm{N} v \lambda 1240$ emission is often very weak and sometimes undetectable (see, e.g., De Breuck et al. 2000). Therefore we developed a new diagnostic diagram for $Z_{\mathrm{NLR}}$ that consists of emission-line flux ratios of $\mathrm{C}$ IV/He II and $\mathrm{C}$ III]/C IV, based on extensive calculations of photoionization models using CLOUDY (Ferland et al. 1998). The reason why this combination of the two emission-line flux ratios works well as a diagnostic of $Z_{\mathrm{NLR}}$ is because the emissivity of CIV $\lambda 1549$ is very sensitive to the gas temperature as it is collisionally excited emission line. Since the equilibrium temperature of NLR clouds is a strong function of $Z_{\mathrm{NLR}}$, higher $Z_{\mathrm{NLR}}$ results in weaker C IV $\lambda 1549$ flux. On the other hand, the emissivity of He II $\lambda 1640$ is almost independent of the gas temperature (and accordingly of $Z_{\mathrm{NLR}}$ ) because it is a recombination line. The flux ratio of $\mathrm{C}$ IV/He II is therefore sensitive to $Z_{\mathrm{NLR}}$. However this flux ratio depends also on other parameters such as the ionization degree of gas and the electron density, as well as $Z_{\mathrm{NLR}}$. The ionization effect is corrected by using $\mathrm{C} \mathrm{III]} / \mathrm{C}$ IV , and finally the diagram of $\mathrm{C}$ IV/He II vs 
C III]/C IV can be used to determine $Z_{\mathrm{NLR}}$ (see Nagao et al. 2006a and Matsuoka et al. 2009 for more details).

Photoionization models and observational results are compared in this diagnostic diagram (Figure 3). In this figure, predictions by shock models (both pure-shock models and shock plus precursor models; Allen et al. 2008) are also shown and we immediately see that the observed flux ratios are well described by photoionization models, but not by shock models. The observed data plotted in this figure have been compiled by Nagao et al. (2006a) and consist of HzRGs at $1.2<z<3.8$, but mainly at $z<2.8$, and newly obtained spectra with VLT/FORS by Matsuoka et al. (2009) that consists of 9 HzRGs at $z>2.7$. There is no systematic difference in the data distribution on the diagnostic diagram between the sample of Nagao et al. (2006a) and that of Matsuoka et al. (2009), suggesting that there is no significant redshift evolution in $Z_{\mathrm{NLR}}$ at $1<z<4$, at least for HzRGs. This result is presented more evidently in Figure 4, where the representative

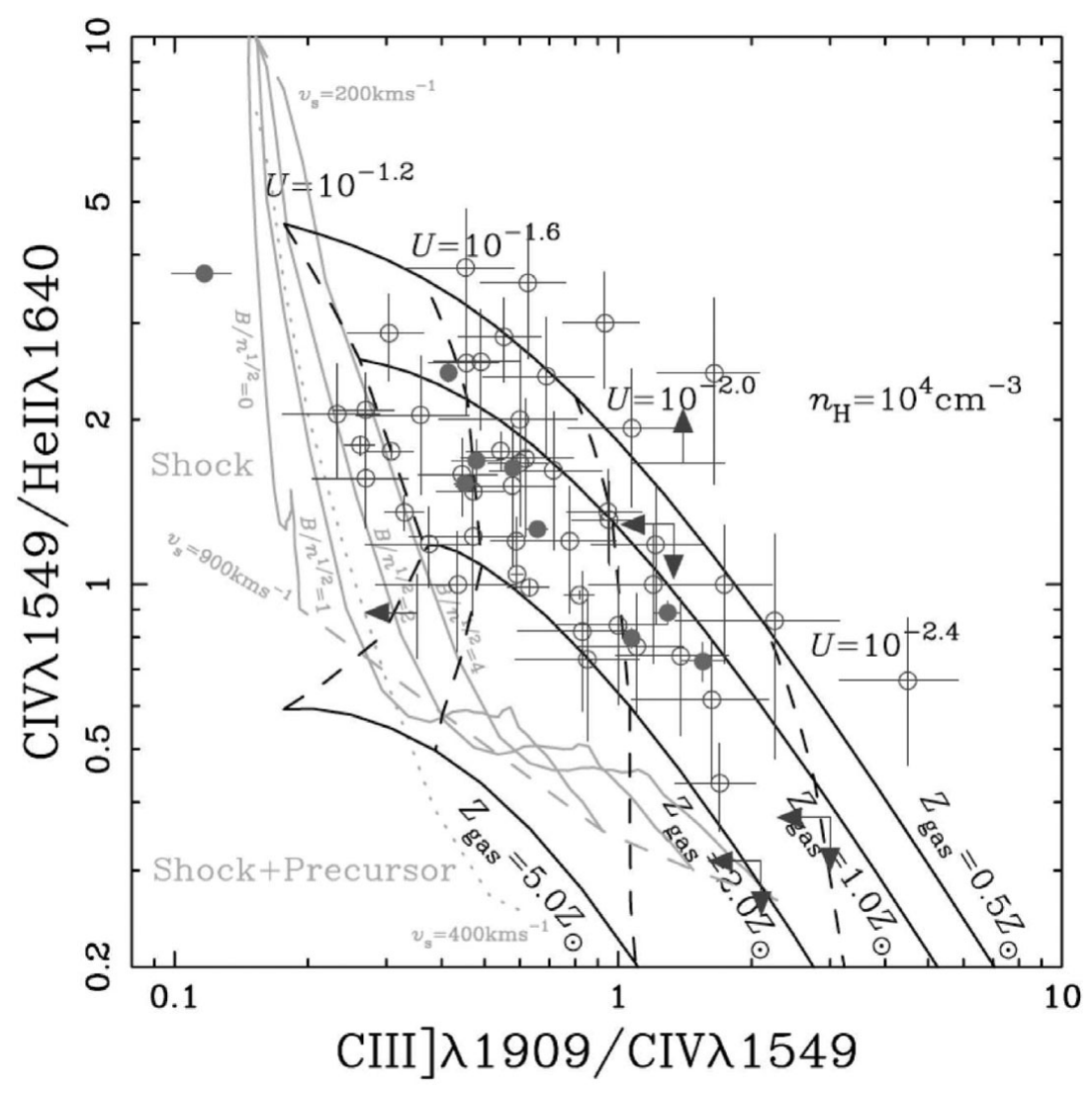

Figure 3. A new metallicity diagnostic diagram that consists of C IV/He II and C III]/C IV. Lines are predictions of photoionization models (labeled by $Z_{\text {gas }}$ ) and shock models (labeled with shock velocity). Open circles with error bars are the data compiled by Nagao et al. (2006a), that are HzRGs at $1.5<z<3.7$ but mainly at $z<2.8$. Filled circles are the data newly obtained with VLT/FORS by Matsuoka et al. (2009), that are HzRGs at $z>2.7$. 
$Z_{\mathrm{NLR}}$ of these HzRG samples is shown as a function of redshift and $L_{\mathrm{AGN}} \dagger$. It is apparent in this figure that $Z_{\mathrm{NLR}}$ shows no significant redshift evolution up to $z \sim 4$. Instead there is a positive correlation between $Z_{\mathrm{NLR}}$ and $L_{\mathrm{AGN}}$, which is nicely consistent with the results obtained for BLRs.

\section{Discussion}

Here we summarize the observational results on AGN metallicity:

- There is a clear positive correlation between $L_{\mathrm{AGN}}$ and AGN metallicity.

- This is mainly due to the dependence of the metallicity on $M_{\mathrm{BH}}$ rather than $L / L_{\mathrm{Edd}}$.

- This correlation shows no significant redshift evolution, probably up to $z \sim 6$.

- These results are consistently seen in both BLRs in SDSS QSOs and NLRs in HzRGs. The most natural interpretation for the origin of the correlation between the AGN metallicity and $M_{\mathrm{BH}}$ is the well-known galaxy mass-metallicity relation (e.g., Tremonti et al. 2004). This is because $M_{\mathrm{BH}}$ is tightly correlated with the mass of spheroidal components, and because metals on both the galactic scale and the AGN scale are originally from the past star-formation activity in host galaxies.

The lack of evolution of the metallicity $-L_{\mathrm{AGN}}$ relation implies that the observed QSOs are chemically mature already, and that the major star formation in QSO host galaxies has been completed at much higher redshift. This would be a challenging issue if the lack of evolution in the metallicity $-L_{\mathrm{AGN}}$ relation corresponds to the lack of evolution of the carbon relative abundance. Since carbon is mostly produced by AGB stars and planetary nebulae, the carbon enhancement requires a relatively long timescale $(\sim 0.5-1 \mathrm{Gyr})$. Since the age of the universe at $z>6$ is less than $1 \mathrm{Gyr}$, it is a puzzling that we do not see "chemically evolving" systems at such high redshifts.

It is also interesting to focus on the lack of evolution of the metallicity $-L_{\mathrm{AGN}}$ relation at lower redshifts, say $2<z<3$. This is because the galaxy mass-metallicity relation shows significant redshift evolution in the redshift range $0<z<3$ (e.g., Maiolino et al. 2008). This suggests that normal galaxies are not mature at this redshift range, which contradicts the observational results seen in AGNs (i.e., AGN host galaxies). Groves et al.

$\dagger$ Since we cannot see directly the (unobscured) AGN continuum emission for type 2 AGNs, we use $L($ He II $\lambda 1640)$ as a measure of $L_{\mathrm{AGN}}$. Note that $L($ He II $\lambda 1640)$ should be proportional to the luminosity of $\mathrm{He}^{+}$-ionizing photons because $\mathrm{He}$ II is a recombination line. See Nagao et al. (2006a) and Matsuoka et al. (2009) for more details.

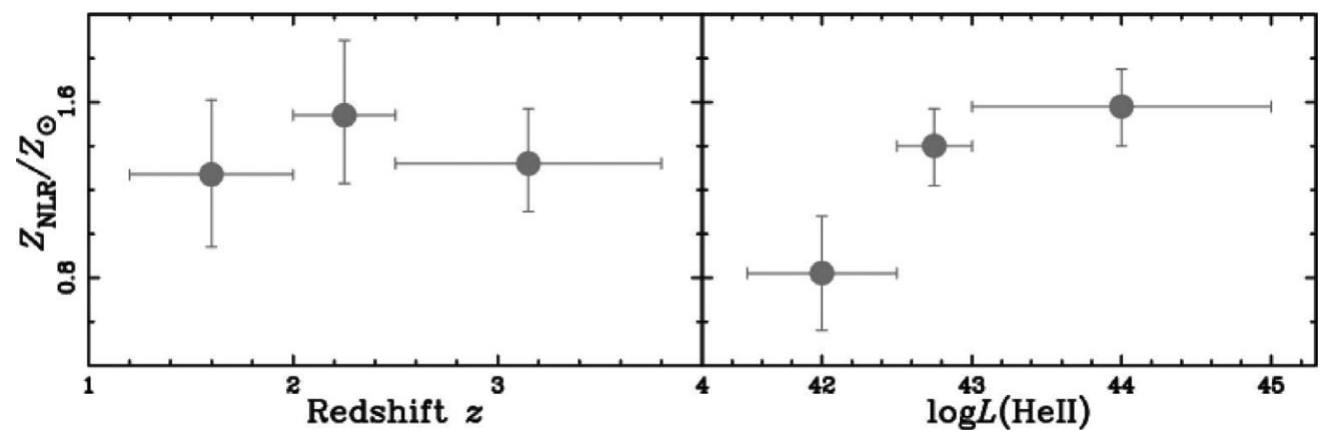

Figure 4. Metallicity of NLRs in HzRGs, as functions of redshift and AGN luminosity (see Matsuoka et al. 2009 for more details). Here $L$ (He II) is used as $L_{\mathrm{AGN}}$, because direct (unabsorbed) emission is not observable for type 2 AGNs. 
(2006) also reported that there are few AGNs with low (sub-solar) metallicity. Why we do not see chemically evolving AGNs at any redshift?

One possible idea to reconcile this apparent contradiction is that we see only mature systems when we search for high-redshift QSOs due to selection effects. As theoretically predicted by Kawakatu et al. (2003) and Granato et al. (2004), QSOs reach their maximum luminosity at the final stage of their evolutionary history, where their chemical properties are expected to be mature. Therefore we have to carry out deeper QSO surveys than SDSS to search for fainter QSOs that may be in an evolving phase of their chemical evolution (Juarez et al. 2009). Note that evolving QSOs are accompanied by active star formation, and thus such QSOs may be obscured by dust. As a result, we could be missing evolving QSOs (Kawakatu et al. 2003, 2008). Next-generation QSO surveys with deeper limiting magnitudes will identify such evolving QSOs (with low luminosity, probably).

\section{References}

Allen, M. G., et al. 2008, ApJS, 178, 20

De Breuck, C., et al. 2000, A\&A A, 362, 519

Dietrich, M., et al. 2003a, ApJ, 596, 817

Dietrich, M., et al. 2003b, ApJ, 589, 722

Ferland, G. J., et al. 1998, PASP, 110, 761

Franceschini, A., et al. 1999, MNRAS, 310, L5

Granato, G. L., et al. 2004, ApJ, 600, 580

Groves, B. A., Heckman, T. M., \& Kauffmann, G. 2006, MNRAS, 371, 1559

Hamann, F. \& Ferland, G. 1993, ApJ, 418, 11

Hamann, F. \& Ferland, G. 1999, ARAA, 37, 487

Imanishi, M. 2002, ApJ, 569, 44

Iwamuro, F., et al. 2004, ApJ, 614, 69

Jiang, L., et al. 2007, AJ, 134, 1150

Juarez, Y., et al. 2009, A\& A, 494, L25

Kawakatu, N., Umemura, M., \& Mori, M. 2003, ApJ, 583, 85

Kawakatu, N. \& Wada, K. 2008, ApJ, 681, 73

Maiolino, R., et al. 2003, ApJ, 596, L155

Maiolino, R., et al. 2008, A\&A, 488, 463

Maiolino, R., et al. 2004, A\&A, 420, 889

Marconi, A. \& Hunt, L. K. 2003, ApJ, 589, L21

Matsuoka, K., et al. 2010, in preparation

Matsuoka, K., et al. 2009, A\&A, 503, 721

Murayama, T., et al. 1998, AJ, 115, 2237

Murayama, T., et al. 1999, AJ, 117, 1645

Nagao, T., Maiolino, R., \& Marconi, A. 2006a, A\&SA, 447, 863

Nagao, T., Marconi, A., \& Maiolino, R. 2006b, A\& $A, 447,157$

Overzier, E. A., et al. 2001, A\&A, 367, L5

Sameshima, H., et al. 2009, MNRAS, 395, 1087

Shemmer, O., et al. 2004, ApJ, 614, 547

Silverman, J. D., et al. 2009, ApJ, 696, 396

Tremonti, C. A., et al. 2004, ApJ, 613, 898

Vestergaard, M. \& Wilkes, B. J. 2001, ApJS, 134, 1

Warner, C., Hamann, F., \& Dietrich, M. 2004, ApJ, 608, 136 